\title{
THE FUNDAMENTAL SOLUTIONS FOR FRACTIONAL EVOLUTION EQUATIONS OF PARABOLIC TYPE
}

\author{
MAHMOUD M. EL-BORAI
}

Received 19 November 2003 and in revised form 22 May 2004

The fundamental solutions for linear fractional evolution equations are obtained. The coefficients of these equations are a family of linear closed operators in the Banach space. Also, the continuous dependence of solutions on the initial conditions is studied. A mixed problem of general parabolic partial differential equations with fractional order is given as an application.

\section{Introduction}

In this paper, we consider the fractional integral evolution equation

$$
u(t)=u_{0}-\frac{1}{\Gamma(\alpha)} \int_{0}^{t}(t-\theta)^{\alpha-1}[A(\theta) u(\theta)-f(\theta)] d \theta
$$

where $0<\alpha \leq 1, \Gamma(\alpha)$ is the gamma function, $\{A(t): t \in[0, T]\}$ is a family of linear closed operators defined on dense set $D(A)$ in a Banach space $E$ into $E, u$ is the unknown $E$ valued function, $u_{0} \in D(A)$, and $f$ is a given $E$-valued function defined on $[0, T]$. It is assumed that $D(A)$ is independent of $t$. Let $B(E)$ denote the Banach space of all linear bounded operators in $E$ endowed with the topology defined by the operator norm. We need the following conditions.

$\left(A_{1}\right)$ The operator $[A(t)+\lambda I]^{-1}$ exists in $B(E)$ for any $\lambda$ with $\operatorname{Re} \lambda \geq 0$ and

$$
\left\|[A(t)+\lambda I]^{-1}\right\| \leq \frac{C}{|\lambda|+1},
$$

for each $t \in[0, T]$, where $C$ is a positive constant independent of both $t$ and $\lambda$.

$\left(A_{2}\right)$ For any $t_{1}, t_{2}, s \in[0, T]$,

$$
\left\|\left[A\left(t_{2}\right)-A\left(t_{1}\right)\right] A^{-1}(s)\right\| \leq C\left|t_{2}-t_{1}\right|^{\gamma},
$$

where $0<\gamma \leq 1, C>0$, and the constants $C$ and $\gamma$ are independent of $t_{1}, t_{2}$, and $s$. 
$\left(A_{3}\right)$ The function $f$ satisfies a uniform Hölder condition (with exponent $\beta$ ) in $[0, T]$, that is,

$$
\left\|f\left(t_{2}\right)-f\left(t_{1}\right)\right\| \leq C\left|t_{2}-t_{1}\right|^{\beta}
$$

for all $t_{1}, t_{2} \in[0, T]$, where $C$ and $\beta$ are positive constants and $0<\beta \leq 1$ (the constants $C$ and $\beta$ are independent of $t_{1}$ and $t_{2}$ ).

Under condition $\left(A_{1}\right)$, each operator $-A(s), s \in[0, T]$, generates an analytic semigroup $\exp (-t A(s)), t>0$, and there exists a positive constant $C$ independent of both $t$ and $s$ such that

$$
\left\|A^{n}(s) \exp (-t A(s))\right\| \leq \frac{C}{t^{n}}
$$

where $n=0,1, t>0, s \in[0, T][9,10]$.

In Section 2, we will construct the fundamental solution of the homogeneous fractional differential equation

$$
\frac{d^{\alpha} v(t)}{d t^{\alpha}}+A(t) v(t)=0, \quad t>0
$$

We will prove the existence and uniqueness of the solution of (1.6), with the initial condition

$$
v(0)=u_{0} \in D(A)
$$

The continuous dependence of the solutions of (1.1) on the elements $u_{0}$ and the function $f$ is proved.

In Section 3, we give an application to a mixed problem of a parabolic partial differential equation of fractional order.

\section{The fundamental solution}

We say that $u$ is a strong solution of the fractional integral equation (1.1) if $u(t) \in D(A)$ for each $t \in[0, T], u, u^{*}$ are continuous in $t \in[0, T]$, and $u$ satisfies $(1.1)$, where $u^{*}(t)=$ $A(t) u(t)$.

Let $h$ be an $E$-valued function defined on $[0, T]$. If $d h(t) / d t$ and the integral $\int_{\tau}^{t}(t-$ $\theta)^{-\alpha}(d h(\theta) / d \theta) d \theta$ exist in the sense of Bochner, then we use the following definition of the fractional derivative ${ }_{\tau} D_{t}^{\alpha} h(t)$ :

$$
{ }_{\tau} D_{t}^{\alpha} h(t)=\frac{1}{\Gamma(1-\alpha)} \int_{\tau}^{t}(t-\theta)^{-\alpha} \frac{d h(\theta)}{d \theta} d \theta
$$

(see $[7,13,16])$.

If $u$ is a strong solution of (1.1), then the fractional derivative

$$
\frac{d^{\alpha} u}{d t^{\alpha}}={ }_{0} D_{t}^{\alpha} u
$$


exists and is continuous in $t \in[0, T]$. In this case, we notice that

$$
\frac{d}{d t} \int_{0}^{t}(t-\theta)^{-\alpha} F(\theta) d \theta=\int_{0}^{t}(t-\theta)^{-\alpha} \frac{d F(\theta)}{d \theta} d \theta
$$

where

$$
F(t)=\int_{0}^{t}(t-\theta)^{\alpha-1}\left[f(\theta)-u^{*}(\theta)\right] d \theta
$$

Using (1.1), (2.1), and (2.3), we get

$$
\begin{aligned}
\frac{d^{\alpha} u(t)}{d t^{\alpha}} & =\frac{1}{\Gamma(\alpha) \Gamma(1-\alpha)} \frac{d}{d t} \int_{0}^{t} \int_{\theta}^{t}(t-s)^{-\alpha}(s-\theta)^{\alpha-1}\left(f(\theta)-u^{*}(\theta)\right) d s d \theta \\
& =-A(t) u(t)+f(t)
\end{aligned}
$$

The converse is also true. In other words, if $d^{\alpha} u(t) / d t^{\alpha}$ is continuous in $t \in[0, T]$ and $u$ represents a solution of the Cauchy problem (2.5), (2.6), then $u$ represents a strong solution of (1.1) (this means that the integral equation (1.1) is equivalent to the Cauchy problem (2.5), (2.6)).

We will consider integrals of operator-valued functions. We denote by $\psi(t, s)$ the integral

$$
\psi(t, s)=\alpha \int_{0}^{\infty} \theta t^{\alpha-1} \zeta_{\alpha}(\theta) \exp \left(-t^{\alpha} \theta A(s)\right) d \theta
$$

where $\zeta_{\alpha}$ is a probability density function defined on $[0, \infty)$ such that its Laplace transform is given by

$$
\int_{0}^{\infty} e^{-\theta x} \zeta_{\alpha}(\theta) d \theta=\sum_{j=0}^{\infty} \frac{(-x)^{j}}{\Gamma(1+\alpha j)},
$$

where $0<\alpha \leq 1, x>0$ [3]. More details about this probability density function can be found in $[8]$.

Lemma 2.1. The improper integral $\int_{0}^{\infty} \theta \zeta_{\alpha}(\theta) A(t) \exp \left(-\eta^{\alpha} \theta A(s)\right) d \theta$ exists for $\eta>0, t, s \in$ $[0, T]$, and represents a uniformly continuous function in the uniform topology (i.e., in the norm of $B(E))$ in the variables $t, \eta, s$, where $t, s \in[0, T], \epsilon \leq \eta \leq T$, and $\epsilon$ is any positive number.

Proof. The existence of the considered improper integral is clear for $\eta>0, t, s \in[0, T]$. If

$$
\begin{aligned}
& 0 \leq t_{1}<t_{1}+\Delta t_{1}=t_{2} \leq T, \\
& \epsilon \leq \eta_{1}<\eta_{1}+\Delta \eta_{1}=\eta_{2} \leq T, \\
& 0 \leq s_{1}<s_{1}+\Delta s_{1}=s_{2} \leq T,
\end{aligned}
$$


then

$$
\begin{aligned}
\int_{0}^{\infty} \theta \zeta_{\alpha} & (\theta) A\left(t_{2}\right) \exp \left(-\eta_{2}^{\alpha} \theta A\left(s_{2}\right)\right) d \theta-\int_{0}^{\infty} \theta \zeta_{\alpha}(\theta) A\left(t_{1}\right) \exp \left(-\eta_{1}^{\alpha} \theta A\left(s_{1}\right)\right) d \theta \\
= & \int_{0}^{\infty} \theta \zeta_{\alpha}(\theta) P\left(t_{1}, t_{2}, s_{2}\right) A\left(s_{2}\right) \exp \left(-\eta_{2}^{\alpha} \theta A\left(s_{2}\right)\right) d \theta \\
& +\int_{0}^{\infty} \theta \zeta(\theta)_{\alpha} A\left(t_{1}\right)\left[\exp \left(-\nu_{2} \theta A\left(s_{2}\right)\right)-\exp \left(-\nu_{1} \theta A\left(s_{2}\right)\right)\right] \exp \left(-\nu_{1} \theta A\left(s_{2}\right)\right) d \theta \\
& +\int_{0}^{\infty} \theta \zeta_{\alpha}(\theta) A\left(t_{1}\right)\left[\exp \left(-\eta_{1}^{\alpha} \theta A\left(s_{2}\right)\right)-\exp \left(-\eta_{1}^{\alpha} \theta A\left(s_{1}\right)\right)\right] d \theta,
\end{aligned}
$$

where $P\left(t_{1}, t_{2}, t_{3}\right)=\left[A\left(t_{2}\right)-A\left(t_{1}\right)\right] A^{-1}\left(t_{3}\right), v_{1}=\eta_{1}^{\alpha} / 2$, and $\nu_{2}=\eta_{2}^{\alpha}-\eta_{1}^{\alpha} / 2$.

It can be proved under conditions $\left(A_{1}\right)$ and $\left(A_{2}\right)$ that

$$
\begin{gathered}
\|A(t)[\exp (-\eta A(s))-\exp (-\eta A(\tau))]\| \leq \frac{C}{\eta}|s-\tau|^{\gamma}, \\
\left\|A(t)[\exp (-\eta A(s))-\exp (-\tau A(s))] A^{-1}(s)\right\| \leq \frac{C|\eta-\tau|}{\operatorname{Min}(\eta, \tau)},
\end{gathered}
$$

for all $\eta>0, \tau>0, t, s \in[0, t]$, where the positive constant $C$ is independent of $t, s, \eta$, and $\tau$. We estimate the norm of the first term on the right-hand side of (2.10) by using condition $\left(A_{2}\right)$ and (1.5). We estimate also the norms of the second term and the last term of the right-hand side of (2.10) by using (2.12), (1.5), and (2.11), respectively. We thus find that the norm of the left-hand side of (2.10) is bounded by

$$
C\left[\frac{\left(\Delta t_{1}\right)^{\gamma}}{\epsilon^{\alpha}}+\frac{1}{\epsilon^{2 \alpha}}\left\{\left(\eta_{1}+\Delta \eta_{1}\right)^{\alpha}-\eta_{1}^{\alpha}\right\}+\epsilon^{1-\alpha}\left(\Delta s_{1}\right)^{\gamma}\right] .
$$

This completes the proof.

Corollary 2.2. The operator-valued functions $\psi(t-\eta, \eta)$ and $A(t) \psi(t-\eta, \eta)$ are continuous in the uniform topology in the variables $t$, $\eta$, where $0 \leq \eta \leq t-\epsilon, 0 \leq t \leq T$, for any $\epsilon>0$. Clearly,

$$
\|\psi(t-\eta, \eta)\| \leq C(t-\eta)^{\alpha-1}
$$

where $C$ is a positive constant independent of $t, \eta$.

LEMMA 2.3. If

$$
w_{1}(t, \tau)=\int_{\tau}^{t} \psi(t-\eta, \eta) f(\eta) d \eta, \quad t>\tau
$$

then

$$
{ }_{\tau} D_{t}^{\alpha} w_{1}(t, \tau)=f(t)-\int_{\tau}^{t} A(\eta) \psi(t-\eta, \eta) f(\eta) d \eta
$$


Proof. Let $\left\{f_{n}\right\}$ be a sequence of functions defined by

$$
f_{n}(t)=\left[I+\frac{1}{n} A(t)\right]^{-1} f(t), \quad t \in[0, T], n=1,2, \ldots
$$

We consider the integrals

$$
\begin{aligned}
& w_{1 n}(t, \tau)=\int_{\tau}^{t} \psi(t-\eta, \eta) f_{n}(\eta) d \eta \\
& w_{2 n}(t, \eta)=f_{n}(\eta)-\frac{1}{\Gamma(\alpha)} \int_{\eta}^{t}(t-\theta)^{\alpha-1} A(\eta) w_{2 n}(\theta, \eta) d \theta
\end{aligned}
$$

Since $f_{n}(t) \in D(A)$ for all $t \in[0, T]$, it follows from [9] that

$$
w_{2 n}(t, \eta)=\int_{0}^{\infty} \zeta_{\alpha}(\theta)\left[\exp \left(-(t-\eta)^{\alpha} \theta A(\eta)\right)\right] f_{n}(\eta) d \theta
$$

where $0 \leq \eta \leq t$.

Thus

$$
\begin{aligned}
{ }_{\eta} D_{t}^{\alpha} w_{2 n}(t, \eta) & =\frac{1}{\Gamma(1-\alpha)} \int_{\eta}^{t}(t-s)^{-\alpha} \frac{d w_{2 n}(s, \eta)}{d s} d s \\
& =\frac{-\alpha}{\Gamma(1-\alpha)} \int_{\eta}^{t} \int_{0}^{\infty}(t-s)^{-\alpha}(s-\eta)^{\alpha-1} \theta \zeta_{\alpha}(\theta) A(\eta) \\
& \times\left[\exp \left(-(s-\eta)^{\alpha} \theta A(\eta)\right)\right] f_{n}(\eta) d \theta d s \\
& =-A(\eta) w_{2 n}(t, \eta) .
\end{aligned}
$$

Using (2.19) and (2.20), we get

$$
\begin{aligned}
{ }_{\tau} D_{t}^{\alpha} \omega_{n}(t, \tau) & =\frac{d}{d t} \int_{\tau}^{t} \int_{0}^{\infty} \zeta_{\alpha}(\theta)\left[\exp \left(-(t-\eta)^{\alpha} \theta A(\eta)\right)\right] f_{n}(\eta) d \theta d \eta \\
& =f_{n}(t)-\int_{\tau}^{t} A(\eta) \psi(t-\eta, \eta) f_{n}(\eta) d \eta
\end{aligned}
$$

According to Lemma 2.1, we notice that $A(\eta) \psi(t-\eta, \eta)$ is a uniformly continuous function in the uniform topology in variables $t, \eta \in[0, T]$, where $t-\eta \geq \epsilon$. Since $f$ satisfies condition $\left(A_{3}\right)$, it follows that the integral $\int_{\tau}^{t} A(\eta) \psi(t-\eta, \eta) f(\eta) d \eta$ exists (cf. [2]).

We notice that

$$
\|A(\eta) \psi(t-\eta, \eta)\| \leq \frac{C}{t-\eta}
$$

for all $t, \eta \in[0, T], t-\eta \geq \epsilon$. Clearly,

$$
\left\|\left[I+\frac{1}{n} A(t)\right]^{-1}-I\right\| \leq C+1,
$$


whereas for $x \in D(A)$,

$$
\left\|\left[I+\frac{1}{n} A(t)\right]^{-1} x-x\right\| \leq \frac{C}{n}\|A(t) x\| .
$$

Using (2.23) and noticing that $f$ satisfies condition $\left(A_{3}\right)$, we deduce that the sequence $\left\{f_{n}\right\}$ uniformly converges to $f$ with respect to $t \in[0, T]$. Using $(2.22)$, we get, for any positive number $\epsilon$, the inequality

$$
\left\|\int_{\tau}^{t-\epsilon} A(\eta) \psi(t-\eta, \eta)\left[f_{n}(\eta)-f(\eta)\right] d \eta\right\| \leq C \epsilon[\ln (t-\tau)-\ln \epsilon],
$$

for a sufficiently large $n$. Consequently,

$$
\lim _{n \rightarrow \infty} D_{t}^{\alpha} w_{1 n}(t, \tau)=f(t)-\int_{\tau}^{t} A(\eta) \psi(t-\eta, \eta) f(\eta) d \eta
$$

uniformly with respect to $t \in[0, T], t>\tau$.

This completes the proof.

Let

$$
\begin{aligned}
\varphi_{1}(t, \tau) & =[A(t)-A(\tau)] \psi(t-\tau, \tau), \\
\varphi_{k+1}(t, \tau) & =\int_{\tau}^{t} \varphi_{k}(t, s) \varphi_{1}(s, \tau) d s, \quad k=1,2, \ldots
\end{aligned}
$$

Using condition $\left(A_{2}\right)$, we get

$$
\left\|\varphi_{1}(t, \tau)\right\| \leq \int_{0}^{\infty}\|S(t, \tau, \theta)\|\left\|\mid A(\tau) \exp \left(-(t-\tau)^{\alpha} \theta A(\tau)\right)\right\| d \theta \leq C(t-\tau)^{\gamma-1},
$$

where

$$
S(t, \tau, \theta)=\alpha \theta(t-\tau)^{\alpha-1} \zeta_{\alpha}(\theta) P(t, \tau, \tau)
$$

Using Lemma 2.1, we conclude that $\varphi_{1}$ is uniformly continuous in $t, \tau$ in the uniform topology provided that $t-\tau \geq \epsilon>0$. Now one verifies, by induction, that all the functions $\varphi_{k}, k=1,2, \ldots$, are uniformly continuous in $t, \tau$ in the uniform topology for $t-\tau \geq \epsilon$, $t, \tau \in[0, T]$, and

$$
\left\|\varphi_{k}(t, \tau)\right\| \leq \frac{C^{k}(t-\tau)^{\gamma k-1}}{\Gamma(\gamma k)}
$$

Using inequalities (2.30), one can justify the relation

$$
\int_{\tau}^{t} \varphi(t, s) \varphi_{1}(s, \tau) d s=\sum_{k=1}^{\infty} \int_{\tau}^{t} \varphi_{k}(t, s) \varphi_{1}(s, \tau) d s
$$


where

$$
\varphi(t, \tau)=\sum_{k=1}^{\infty} \varphi_{k}(t, \tau) .
$$

It is easy to see that

$$
\|\varphi(t, \tau)\| \leq C(t-\tau)^{\gamma-1} .
$$

The function $\varphi$ is uniformly continuous in the uniform topology in $t, \tau$ provided that $0 \leq \tau \leq t-\epsilon, \epsilon \leq t \leq T$ for any $\epsilon>0$. Using Fubini's theorem, we deduce that $\varphi$ is the unique solution of the integral equation

$$
\varphi(t, \tau)=\varphi_{1}(t, \tau)+\int_{\tau}^{t} \varphi(t, s) \varphi_{1}(s, \tau) d s
$$

Lemma 2.4. For any $0<\delta<\gamma, 0 \leq \tau<t_{1}<t_{2} \leq T$,

$$
\left\|\varphi\left(t_{2}, \tau\right)-\varphi\left(t_{1}, \tau\right)\right\| \leq C\left(t_{2}-t_{1}\right)^{\gamma-\delta}\left(t_{1}-\tau\right)^{\delta-1}
$$

where the positive constant $C$ does not depend on $t_{1}, t_{2}$, or $\tau$.

Proof. From (2.28), we get

$$
\left\|\varphi_{1}\left(t_{2}, \tau\right)-\varphi_{1}\left(t_{1}, \tau\right)\right\| \leq 2 C\left(t_{1}-\tau\right)^{\gamma-1} .
$$

Writing

$$
\begin{aligned}
\varphi_{1}\left(t_{2}, \tau\right)-\varphi_{1}\left(t_{1}, \tau\right)= & P\left(t_{1}, t_{2}, \tau\right) A(\tau) \psi\left(t_{2}-\tau, \tau\right) \\
& +P\left(t_{1}, \tau, \tau\right) A(\tau)\left[\psi\left(t_{2}-\tau, \tau\right)-\psi\left(t_{1}-\tau, \tau\right)\right], \\
\Lambda\left(t_{1}, t_{2}, \tau\right)= & P\left(t_{1}, \tau, \tau\right) A(\tau)\left[\psi\left(t_{2}-\tau, \tau\right)-\psi\left(t_{1}-\tau, \tau\right)\right],
\end{aligned}
$$

we get

$$
\begin{gathered}
\left\|P\left(t_{1}, t_{2}, \tau\right) A(\tau) \psi\left(t_{2}-\tau, \tau\right)\right\| \leq C\left(t_{2}-t_{1}\right)^{\gamma}\left(t_{1}-\tau\right)^{-1} \\
\left\|\Lambda\left(t_{1}, t_{2}, \tau\right)\right\| \leq 2 C\left(t_{1}-\tau\right)^{\gamma-1}
\end{gathered}
$$

We can write

$$
\Lambda\left(t_{1}, t_{2}, \tau\right)=\int_{0}^{\infty} P_{1}\left(t_{1}, t_{2}, t_{3}, \tau, \theta\right) d \theta+\int_{0}^{\infty} P_{2}\left(t_{1}, t_{2}, t_{3}, \tau, \theta\right) d \theta,
$$

where

$$
\begin{aligned}
P_{1}\left(t_{1}, t_{2}, \tau, \theta\right) & =\alpha \theta \zeta_{\alpha}(\theta)\left[A\left(t_{1}\right)-A(\tau)\right] P_{3}\left(t_{1}, t_{2}, t_{3}, \tau, \theta\right) \exp \left[-\left(t_{1}-\tau\right)^{\alpha} \theta A(\tau)\right], \\
P_{3}\left(t_{1}, t_{2}, t_{3}, \tau, \theta\right) & =\left(t_{2}-\tau\right)^{\alpha-1} \exp \left[-\left\{\left(t_{2}-\tau\right)^{\alpha}-\left(t_{1}-\tau\right)^{\alpha}\right\} \theta A(\tau)\right]-\left(t_{2}-\tau\right)^{\alpha-1} I .
\end{aligned}
$$


We can find $t_{3}$ and $t_{4}$ such that $t_{1}<t_{3}<t_{2}, t_{1}<t_{4}<t_{2}$, and

$$
\begin{aligned}
P_{3}\left(t_{1}, t_{2}, t_{3}, \tau, \theta\right) & =\left(t_{2}-\tau\right)^{\alpha-1} \exp \left[-\alpha\left(t_{2}-t_{1}\right)\left(t_{3}-\tau\right)^{\alpha-1} \theta A(\tau)\right]-\left(t_{2}-\tau\right)^{\alpha-1} I, \\
P_{2}\left(t_{1}, t_{2}, t_{3}, \tau, \theta\right) & =\alpha \theta \zeta_{\alpha}(\theta)\left[A\left(t_{1}\right)-A(\tau)\right]\left[\left(t_{2}-\tau\right)^{\alpha-}-\left(t_{1}-\tau\right)^{\alpha-1}\right] \exp \left[-\left(t_{1}-\tau\right) \theta A(\tau)\right] \\
& =\alpha(\alpha-1) \theta \zeta_{\alpha}(\theta)\left(t_{2}-t_{1}\right)\left(t_{4}-\tau\right)^{\alpha-2}\left[A\left(t_{1}\right)-A(\tau)\right] \exp \left[-\left(t_{1}-\tau\right) \theta A(\tau)\right] .
\end{aligned}
$$

We notice that

$$
P_{3}\left(t_{1}, t_{2}, t_{3}, \tau, \theta\right)=-\alpha \theta\left(t_{2}-\tau\right)^{\alpha-1}\left(t_{3}-\tau\right)^{\alpha-1} \int_{0}^{t_{2}-t_{1}} A(\tau) \exp \left[-\eta \alpha \theta\left(t_{3}-\tau\right)^{\alpha-1} A(\tau)\right] d \eta
$$

Now it is easy to see that

$$
\left\|\Lambda\left(t_{1}, t_{2}, \tau\right)\right\| \leq C\left(t_{1}-\tau\right)^{\gamma-2}\left(t_{2}-t_{1}\right) .
$$

Using the two bounds of $\left\|\Lambda\left(t_{1}, t_{2}, \tau\right)\right\|$, we get

$$
\begin{aligned}
\left\|\Lambda\left(t_{1}, t_{2}, \tau\right)\right\| & =\left\|\Lambda\left(t_{1}, t_{2}, \tau\right)\right\|^{\gamma}\left\|\Lambda\left(t_{1}, t_{2}, \tau\right)\right\|^{1-\gamma} \\
& \leq C\left(t_{1}-\tau\right)^{-1}\left(t_{2}-t_{1}\right)^{\gamma}
\end{aligned}
$$

Consequently,

$$
\left\|\varphi_{1}\left(t_{2}, \tau\right)-\varphi_{1}\left(t_{1}, \tau\right)\right\| \leq C\left(t_{1}-\tau\right)^{-1}\left(t_{2}-t_{1}\right)^{\gamma} .
$$

Using (2.36) and (2.45), we get

$$
\left\|\varphi_{1}\left(t_{2}, \tau\right)-\varphi_{1}\left(t_{1}, \tau\right)\right\|^{\delta_{1}+\delta_{2}} \leq C^{\delta_{1}+\delta_{2}}\left(t_{2}-t_{1}\right)^{\gamma \delta_{1}}\left(t_{1}-\tau\right)^{\delta_{2} \gamma-\delta_{1}-\delta_{2}},
$$

where $\delta_{1}>0, \delta_{2}>0$.

Thus

$$
\left\|\varphi_{1}\left(t_{2}, \tau\right)-\varphi_{1}\left(t_{1}, \tau\right)\right\| \leq C\left(t_{2}-t_{1}\right)^{\gamma-\delta}\left(t_{1}-\tau\right)^{\delta-1},
$$

where $\delta=\delta_{2} \gamma /\left(\delta_{1}+\delta_{2}\right)<\gamma$.

Using (2.34), we get

$$
\begin{aligned}
\varphi\left(t_{2}, \tau\right)-\varphi\left(t_{1}, \tau\right)= & \varphi_{1}\left(t_{2}, \tau\right)-\varphi_{1}\left(t_{1}, \tau\right) \\
& +\int_{\tau}^{t_{1}}\left[\varphi_{1}\left(t_{2}, s\right)-\varphi_{1}\left(t_{1}, s\right)\right] \varphi(s, \tau) d s+\int_{t_{1}}^{t_{2}} \varphi_{1}\left(t_{2}, s\right) \varphi(s, \tau) d s .
\end{aligned}
$$

We estimate the norm of the first term on the right-hand side of (2.48) by using (2.47) and the norm of the second term by using (2.28) and (2.33). After simple calculations, the required result follows. 
We will make use of the inequality

$$
\left\|A(t) A^{-1}(s)\right\| \leq C,
$$

which follows from condition $\left(A_{2}\right)$, where $t, s \in[0, T]$ and $C$ is a positive constant independent of both $t$ and $s$.

THeORem 2.5. There exists an operator-valued function $Q(t)$ in $B(E)$, defined and strongly continuous in $t$ for $0 \leq t \leq T$, such that

$\left(B_{1}\right)$ the fractional derivative $d^{\alpha} Q(t) / d t^{\alpha}$ exists in the strong topology, belongs to $B(E)$ for $0 \leq t \leq T$, and is strongly continuous in $t$ for $0 \leq t \leq T$,

$\left(B_{2}\right)$ the range of $Q(t)$ is included in $D(A)$ for $0 \leq t \leq T$,

$\left(B_{3}\right)$ for any $u_{0} \in E, Q(t) u_{0}$ satisfies the fractional differential equation

$$
\frac{d^{\alpha} Q(t)}{d t^{\alpha}} u_{0}+A(t) Q(t) u_{0}=0, \quad 0<t \leq T
$$

$\left(B_{4}\right) Q(0)=A^{-1}(0)$,

$\left(B_{5}\right)$ a solution of the Cauchy problem (1.6), (1.7) is given by $v(t)=Q(t) A(0) u_{0}$, for any $u_{0} \in D(A)$.

Proof. We set

$$
Q(t)=A^{-1}(0)+\int_{0}^{t} \psi(t-\eta, \eta) U(\eta) d \eta
$$

We will determine the operator-valued function $U(t)$ such that $Q(t) u_{0}$ satisfies (2.50).

Using formally Lemma 2.3, we get

$$
U(t) u_{0}+\int_{0}^{t} \varphi_{1}(t, \eta) U(\eta) u_{0} d \eta=-A(t) A^{-1}(0) u_{0}
$$

(cf. $[11,14,15])$.

The operator-valued function $U(t)$ can be obtained by successive approximations, that is, we put

$$
U(t)=\sum_{k=0}^{\infty} U_{k}(t)
$$

where $U_{0}(t)=-A(t) A^{-1}(0)$,

$$
U_{k+1}(t)=-\int_{0}^{t} \varphi_{1}(t, s) U_{k}(s) d s
$$

Using the properties of $\varphi_{k}$ and Fubini's theorem, one easily shows, by induction, that

$$
U_{k}(t)=-\int_{0}^{t} \varphi_{k}(t, s) A(s) A^{-1}(0) d s .
$$


Using (2.33), (2.54), and (2.55), we deduce that the series $\sum_{k=0}^{\infty} U_{k}(t)$ uniformly converges on $[0, T]$.

It is clear that $U(t)$ is given by

$$
U(t)=-A(t) A^{-1}(0)-\int_{0}^{t} \varphi(t, s) A(s) A^{-1}(0) d s
$$

Using (2.33), we get

$$
\|U(t)\| \leq C+C t^{\gamma}
$$

It is easy to see that

$$
\begin{aligned}
U\left(t_{2}\right)-U\left(t_{1}\right)= & {\left[A\left(t_{1}\right)-A\left(t_{2}\right)\right] A^{-1}(0) } \\
& -\int_{0}^{t_{1}}\left[\varphi\left(t_{2}, s\right)-\varphi\left(t_{1}, s\right)\right] A(s) A^{-1}(0) d s \\
& -\int_{t_{1}}^{t_{2}} \varphi\left(t_{2}, s\right) A(s) A^{-1}(0) d s .
\end{aligned}
$$

Using condition $\left(A_{2}\right)$ and Lemma 2.4, we find that

$$
\left\|U\left(t_{2}\right)-U\left(t_{1}\right)\right\| \leq C\left(t_{2}-t_{1}\right)^{\gamma}+\frac{c}{\delta}\left(t_{2}-t_{1}\right)^{\gamma-\delta} t_{1}^{\delta}+\frac{c}{\gamma}\left(t_{2}-t_{1}\right)^{\gamma}
$$

where $t_{2}>t_{1}, t_{1}, t_{2} \in[0, T]$, and $C$ is a positive constant independent of $t_{1}, t_{2}$.

Recalling that $\psi(t-\eta, \eta)$ is uniformly continuous in $t, \eta$, provided that $t-\eta \geq \epsilon>0$, and using (2.14), (2.59), one can verify without difficulty that $\int_{0}^{t} \psi(t-\eta, \eta) U(\eta) d \eta$ is uniformly continuous (in the norm of $B(E)$ ) in $t \in[0, T]$. Using (2.57), we get $\|Q(t)\| \leq C$ for all $t \in[0, T]$, where $C$ is a positive constant independent of $t$. It is also obvious that $Q(0)=A^{-1}(0)$ and $Q(t) u_{0}$ is contiguous in $t \in[0, T]$ for every $u_{0} \in E$. We prove now that the range of $Q(t)$ is included in $D(A)$ for $0<t \leq T$.

Using (2.59) and Lemma 2.1, we deduce that $A(t) \psi(t-\eta, \eta) U(\eta)$ is uniformly continuous in the uniform topology in the variables $t, \eta \in[0, T]$, provided that $t-\eta \geq \epsilon$, where $\epsilon$ is any positive number.

The operator-valued function $A(t) \psi(t-\eta, \eta) U(\eta)$ can be written in the form

$$
\begin{aligned}
A(t) \psi(t-\eta, \eta) U(\eta)= & A(t)[\psi(t-\eta, \eta)-\psi(t-\eta, t)] U(\eta) \\
& +A(t) \psi(t-\eta, t)[U(\eta)-U(t)]+A(t) \psi(t-\eta, t) U(t) .
\end{aligned}
$$

By using (2.11) and (2.57), we find that the norm of the first term on the right-hand side of (2.60) is bounded by $C(t-\eta)^{\gamma-1}$. By using (1.5) and (2.59), we find that the norm of the second term on the right-hand side of (2.60) is bounded by $C(t-\eta)^{\gamma-\delta-1}$ (where $C$ is a generic positive constant independent of both $t$ and $\eta$ ). Using these estimations and 
noticing that

$$
\begin{aligned}
\int_{0}^{t} A(t) & \psi(t-\eta, \eta) U(\eta) u_{0} d \eta \\
= & \int_{0}^{t} A(t)[\psi(t-\eta, \eta)-\psi(t-\eta, t)] U(\eta) u_{0} d \eta \\
& +\int_{0}^{t} A(t) \psi(t-\eta, t)[U(\eta)-U(t)] u_{0} d \eta \\
& -\int_{0}^{\infty} \zeta_{\alpha}(\theta)\left[\exp \left(-t^{\alpha} \theta A(t)\right)\right] U(t) u_{0} d \theta+U(t) u_{0}
\end{aligned}
$$

one can deduce that the integral $\int_{0}^{t} A(t) \psi(t-\eta, \eta) U(\eta) u_{0} d \eta$ is continuous in $t \in[0, T]$, for every $u_{0} \in E$. Consequently, the range of $Q(t)$ is included in $D(A)$ for every $t \in[0, T]$. It can be proved that there are two positive constants $C$ and $\delta$ such that

$$
\left\|A(t) Q(t) u_{0}\right\| \leq C+C t^{\delta}, \quad t \in[0, T]
$$

where $0<\delta<1$ and $C$ is independent of $t, u_{0} \in E$. Using (2.51), (2.59), and Lemma 2.3, one can easily show that $\left(d^{\alpha} Q(t) / d t^{\alpha}\right) \mathcal{u}_{0}$ exists and represents a continuous function in $t \in[0, T]$ for every $u_{0} \in E$.

It is also clear that $Q(t) u_{0}$ satisfies (2.50). The function $v(t)=Q(t) A(0) u_{0}$ represents a solution of the Cauchy problem (1.6), (1.7) if $u_{0} \in D(A)$. This completes the proof of the properties $B_{1}-B_{5}$.

Theorem 2.6. A solution of the Cauchy problem (2.5), (2.6) is given by

$$
\begin{aligned}
u(t)= & u_{0}+\int_{0}^{t} \psi(t-\eta, \eta) U(\eta) A(0) u_{0} d \eta+\int_{0}^{t} \psi(t-\eta, \eta) f(\eta) d \eta \\
& +\int_{0}^{t} \int_{0}^{\eta} \psi(t-\eta, \eta) \varphi(\eta, s) f(s) d s d \eta
\end{aligned}
$$

or

$$
\begin{aligned}
u(t)= & u_{0}-\int_{0}^{t} \psi(t-\eta, \eta) A(\eta) u_{0} d \eta-\int_{0}^{t} \int_{0}^{\eta} \psi(t-\eta, \eta) \varphi(\eta, s) A(s) u_{0} d s d \eta \\
& +\int_{0}^{t} \psi(t-\eta, \eta) f(\eta) d \eta+\int_{0}^{t} \int_{0}^{\eta} \psi(t-\eta, \eta) \varphi(\eta, s) f(s) d s d \eta,
\end{aligned}
$$

where $u_{0} \in D(A)$ and $f$ satisfies condition $\left(A_{3}\right) ; t \in[0, T]$.

Proof. We set

$$
u(t)=A^{-1}(0) u_{0}+\int_{0}^{t} \psi(t-\eta, \eta) V(\eta) d \eta
$$

Then we determine the function $V$ such that $u$ satisfies (2.5). The proof is carried out similar to that of Theorem 2.5.

Theorem 2.7. The strong solution of the Cauchy problem (1.6), (1.7) is unique. 
Proof. We introduce the bounded operators $A_{n}(t)=A(t)[I+(1 / n) A(t)]^{-1}$. It is known that

$$
\begin{gathered}
\left\|\left(A_{n}(t)-\lambda I\right)^{-1}\right\| \leq \frac{C}{|\lambda|+1}, \\
\left\|\left(A_{n}(t)-A_{n}(\tau)\right) A_{n}^{-1}(s)\right\| \leq C|t-\tau|^{\gamma},
\end{gathered}
$$

where $s, t, \tau \in[0, T]$ and $C$ is a positive constant independent of $t, \tau, s$, and $n$.

Consider the following Cauchy problem:

$$
\begin{gathered}
\frac{d^{\alpha} v_{n}(t)}{d t^{\alpha}}+A_{n}(t) v_{n}(t)=0, \quad n=1,2, \ldots, \\
v_{n}(0)=u_{0} .
\end{gathered}
$$

The function $w_{n}(t)=v(t)-v_{n}(t)$ then satisfies

$$
\begin{aligned}
\frac{d^{\alpha} w_{n}(t)}{d t^{\alpha}}+A_{n}(t) w_{n}(t) & =g_{n}(t), \quad t \in[0, T], \\
w_{n}(0) & =0,
\end{aligned}
$$

where $g_{n}(t)=\left[A_{n}(t)-A(t)\right] v(t)$.

The solution of the Cauchy problem (2.69) is unique. To prove this fact, suppose $g_{n}(t)=0$. Then $w_{n}(t)$ satisfies

$$
\begin{aligned}
\left\|w_{n}(t)\right\| & \leq \frac{1}{\Gamma(\alpha)} \int_{0}^{t}(t-\theta)^{\alpha-1}\left\|A_{n}(\theta) w_{n}(\theta)\right\| d \theta \\
& \leq \frac{C_{n}}{\Gamma(\alpha)} \int_{0}^{t}(t-\theta)^{\alpha-1}\left\|w_{n}(\theta)\right\| d \theta,
\end{aligned}
$$

for every $n$, where $C_{n}$ is a positive constant. It follows that $w_{n}(t)=0$ for all $t \in[0, T]$. Noticing that $g_{n}$ is continuous in $t \in[0, T]$ for every $n=1,2, \ldots$ and $A_{n}(t)$ is a bounded operator that varies continuously in $t \in[0, T]$ (in the uniform topology), then it is easy to see, with the help of (2.5), that the unique solution of the Cauchy problem (2.69) is given by

$$
w_{n}(t)=\int_{0}^{t} \psi_{n}(t-\eta, \eta) g_{n}(\eta) d \eta+\int_{0}^{t} \int_{0}^{\eta} \psi_{n}(t-\eta, \eta) \varphi^{(n)}(\eta, s) g_{n}(s) d s d \eta,
$$

where

$$
\psi_{n}(t-\eta, \eta)=\alpha \int_{0}^{t} \theta(t-\eta)^{\alpha-1} \zeta_{\alpha}(\theta) \exp \left[-(t-\eta)^{\alpha} \theta A_{n}(\eta)\right] d \theta
$$

$\varphi^{(n)}(t, \tau)$ is the unique solution of the integral equation

$$
\begin{aligned}
& \varphi_{1}^{(n)}(t, \tau)=\varphi_{1}^{(n)}(t, \tau)+\int_{\tau}^{t} \varphi^{(n)}(t, s) \varphi_{1}^{(n)}(s, t) d s, \\
& \varphi_{1}^{(n)}(t, \tau)=\left[A_{n}(t)-A_{n}(\tau)\right] \psi_{n}(t-\tau, \tau) .
\end{aligned}
$$


It can be shown that the sequence $\left\{g_{n}\right\}$ uniformly converges to zero in $E$ with respect to $t \in[0, T]$.

Consequently, by using (2.14), (2.33), (2.51), (2.67), and (2.71), we get $v(t)=$ $\lim _{n \rightarrow \infty} v_{n}(t)$ uniformly with respect to $t \in[0, T]$. Since $v_{n}(t)$ is uniquely defined as a solution of the Cauchy problem (2.68), $v(t)$ is also unique.

The continuous dependence of the solution of the Cauchy problem (2.5), (2.6) on $f$ and $u_{0}$ is established from formula (2.64) (cf. [1]).

It must be noticed that the fractional differential equations have many important applications in different areas of applied mathematics (see $[5,6,12])$.

\section{Application}

Let $\Omega$ be a bounded domain in the real $n$-dimensional Euclidean space $R^{n}$. For any $0<$ $T<\infty$, denote by $Q_{T}$ the cylinder $\{(x, t): x \in \Omega, 0<t<T\}$ and by $\partial \Omega$ the boundary of $\Omega$.

We consider the differential operator

$$
\frac{\partial^{\alpha}}{\partial t^{\alpha}}+A^{*}(x, t, D)=\frac{\partial^{\alpha}}{\partial t^{\alpha}}+\sum_{|q| \leq 2 m} a_{q}(x, t) D^{q},
$$

where $A^{*}(x, t, D)$ is said to be uniformly elliptic in $\overline{Q_{T}}$ if the coefficients $a_{q}(x, t)$ are bounded in $\overline{Q_{T}}$ and $(-1)^{m} \operatorname{Re} \sum_{|q|=2 m} a_{q}(x, t) \xi^{q} \geq C|\xi|^{2 m}$, for all $(x, t) \in \overline{Q_{T}}$ and for all real $\xi$, where $C$ is a positive constant independent of $x, t, \xi$, and

$$
\begin{gathered}
|\xi|^{2}=\xi_{1}^{2}+\cdots+\xi_{n}^{2} \quad\left(\overline{Q_{T}}=\{(x, t): x \in \Omega \cup \partial \Omega, 0 \leq t \leq T\}\right) \\
\left(D^{q}=D_{1}^{q_{1}} \cdots D_{n}^{q_{n}}, D_{j}=\frac{\partial}{\partial x_{j}},|q|=q_{1}+\cdots+q_{n}, q=\left(q_{1}, \ldots, q_{n}\right) \text { is a multi-index }\right) .
\end{gathered}
$$

We consider the Cauchy problem of the fractional evolution equation

$$
\begin{gathered}
\frac{d^{\alpha} u}{d t^{\alpha}}+A^{*}(t) u=f(t), \quad 0<t \leq T, \\
u(0)=u_{0},
\end{gathered}
$$

in the Hilbert space $L^{2}(\Omega)$, where for each $t, f(t)$ is the function $f(x, t)$ belonging to $L_{2}(\Omega), A^{*}(t)$ is the operator with domain $D\left(A^{*}\right)=H^{2 m}(\Omega) \cap H_{0}^{m}(\Omega)$ given by $A^{*}(t)=$ $A^{*}(x, t, D)$, and $u_{0}$ is a function in $H^{2 m}(\Omega) \cap H_{0}^{m}(\Omega)$ (see $\left.[2,4]\right)$.

$\left(H^{m}(\Omega)\right)$ is the completion of the space $C^{m}(\Omega)$ with respect to the norm

$$
\|f\|_{m}=\left[\sum_{|q| \leq m} \int_{\Omega}\left[D^{q} f(x)\right]^{2} d x\right]^{1 / 2},
$$

$C^{m}(\Omega)$ is the set of all continuous functions defined on $\Omega$ which have continuous partial derivatives of order less than or equal to $m, H_{0}^{m}(\Omega)$ is the completion of $C_{0}^{m}(\Omega)$ with respect to the norm $\|f\|_{m}$, and $C_{0}^{m}(\Omega)$ is the set of all functions $f \in C^{m}(\Omega)$ with compact supports in $\Omega$. 
It is assumed that

(I) all the coefficients $a_{q}(x, t)$ are continuous in $\overline{Q_{T}}$ and $\left|a_{q}\left(x, t_{2}\right)-a_{q}\left(x, t_{1}\right)\right| \leq$ $C\left|t_{2}-t_{1}\right|^{\gamma}, 0<\gamma \leq 1, t_{1}, t_{2} \in[0, T], C$ is a positive constant independent of $t_{1}$, $t_{2}$, and $x \in \Omega$;

(II) $\left[\int_{\Omega}\left|f\left(x, t_{2}\right)-f\left(x, t_{1}\right)\right|^{2} d x\right]^{1 / 2} \leq C\left|t_{2}-t_{1}\right|^{\beta} ; 0<\beta \leq 1$ and $C$ is a positive constant independent of $t_{1}$ and $t_{2}$.

Theorem 3.1. Assume that $A^{*}(x, t, D)$ is uniformly elliptic in $\overline{Q_{T}}$, that (I) and (II) hold, and that $\partial \Omega$ is of class $C^{2 m}$. Then there exists a unique strong solution of problem (3.3), (3.4).

Proof. Writing (3.3) in the form

$$
\frac{d^{\alpha} u}{d t^{\alpha}}+\left[A^{*}(t)+k I\right] u=f(t)+k u
$$

we see that for some constant $k$, the operator $A^{*}(t)+k I$ satisfies conditions $\left(A_{1}\right)$ and $\left(A_{2}\right)$. Using formula (2.64), we get

$$
\begin{aligned}
u(t)= & u_{0}-\int_{0}^{t} \psi(t-\eta, \eta) A(\eta) u_{0} d \eta-\int_{0}^{t} \int_{0}^{\eta} \psi(t-\eta, \eta) \varphi(\eta, s) A(s) u_{0} d s d \eta \\
& +\int_{0}^{t} \psi(t-\eta, \eta)[f(\eta)+k u(\eta)] d \eta \\
& +\int_{0}^{t} \int_{0}^{\eta} \psi(t-\eta, \eta) \varphi(\eta, s)[f(s)+k u(s)] d s d \eta \\
A(t)= & A^{*}(t)+k I
\end{aligned}
$$

It can be proved that the last integral equation has the unique required solution $u(t)$. This completes the proof.

\section{Acknowledgment}

I would like to thank the referees and Professor Jewgeni H. Dshalalow for their careful reading of the manuscript and their valuable comments.

\section{References}

[1] M. M. El-Borai, Some characterization of an abstract differential equation, Proc. Math. Phys. Soc. Egypt (1979), no. 48, 15-23.

[2] - Some probability densities and fundamental solutions of fractional evolution equations, Chaos Solitons Fractals 14 (2002), no. 3, 433-440.

[3] W. Feller, An Introduction to Probability Theory and Its Applications. Vol. II, John Wiley \& Sons, New York, 1971.

[4] A. Friedman, Partial Differential Equations, Holt, Rinehart and Winston, New York, 1969.

[5] C. Friedrich, Linear viscoelastic behavior of branched polybutadienes: a fractional calculus approach, Acta Polymerica 46 (1995), no. 5, 385-390.

[6] C. Friedrich and H. Braun, Linear viscoelastic behaviour of complex materials: a fractional mode representation, Colloid Polym. Sci. 272 (1994), 1536-1546.

[7] I. M. Gelfand and G. E. Shilov, Generalized Functions. Vol. I: Properties and Operations, Nauka, Moscow, 1959. 
[8] R. Gorenflo and F. Mainardi, Fractional calculus and stable probability distributions, Arch. Mech. (Arch. Mech. Stos.) 50 (1998), no. 3, 377-388.

[9] E. Hille and R. S. Phillips, Functional Analysis and Semi-Groups, American Mathematical Society Colloquium Publications, vol. 31, American Mathematical Society, Rhode Island, 1957.

[10] T. Kato and H. Tanabe, On the abstract evolution equation, Osaka J. Math. 14 (1962), 107-133.

[11] S. Kawatsu, Cauchy problem for abstract evolution equations of parabolic type, J. Math. Kyoto Univ. 30 (1990), no. 1, 59-91.

[12] R. C. Koeller, Applications of fractional calculus to the theory of viscoelasticity, Trans. ASME J. Appl. Mech. 51 (1984), no. 2, 299-307.

[13] W. R. Schneider and W. Wyss, Fractional diffusion and wave equations, J. Math. Phys. 30 (1989), no. $1,134-144$.

[14] P. E. Sobolevski, On equations of parabolic type in a Banach space, Trudy Moscow Math. 10 (1961), 289-350.

[15] H. Tanabe, On regularity of solutions of abstract differential equations of parabolic type in Banach space, J. Math. Soc. Japan 19 (1967), 521-542.

[16] W. Wyss, The fractional diffusion equation, J. Math. Phys. 27 (1986), no. 11, 2782-2785.

Mahmoud M. El-Borai: Department of Mathematics, Faculty of Science, Alexandria University, Alexandria, Egypt

E-mail address: m_m_elborai@yahoo.com 


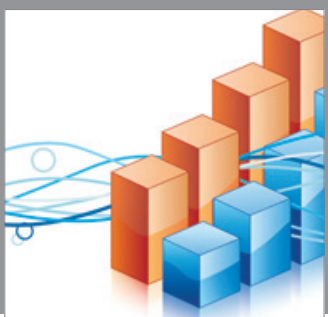

Advances in

Operations Research

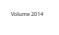

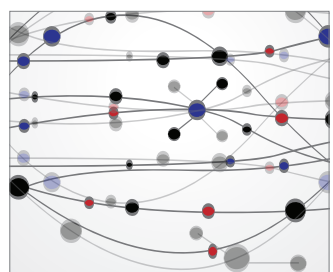

\section{The Scientific} World Journal
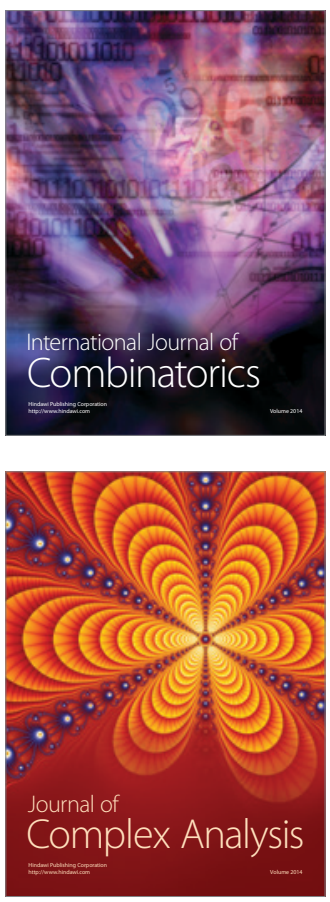

International Journal of

Mathematics and

Mathematical

Sciences
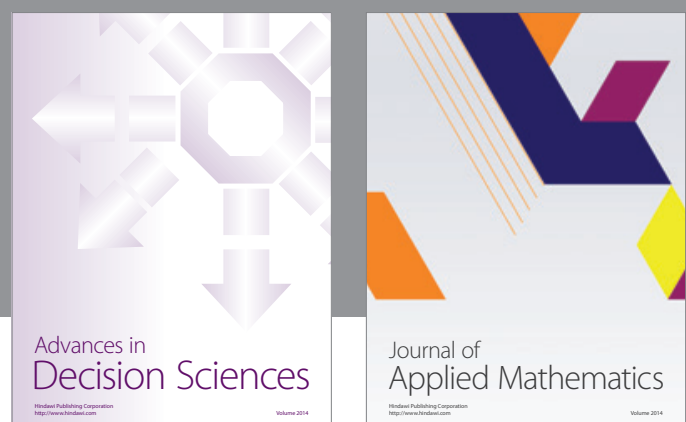

Journal of

Applied Mathematics
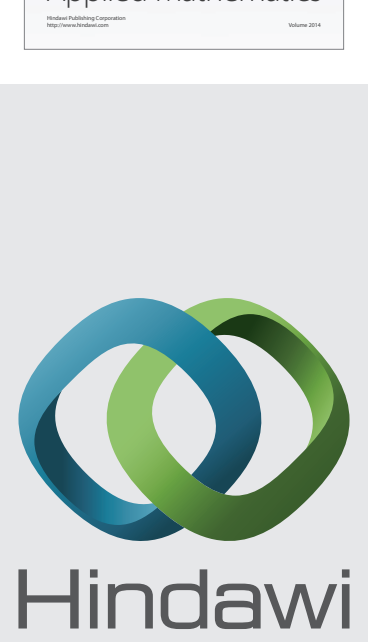

Submit your manuscripts at http://www.hindawi.com
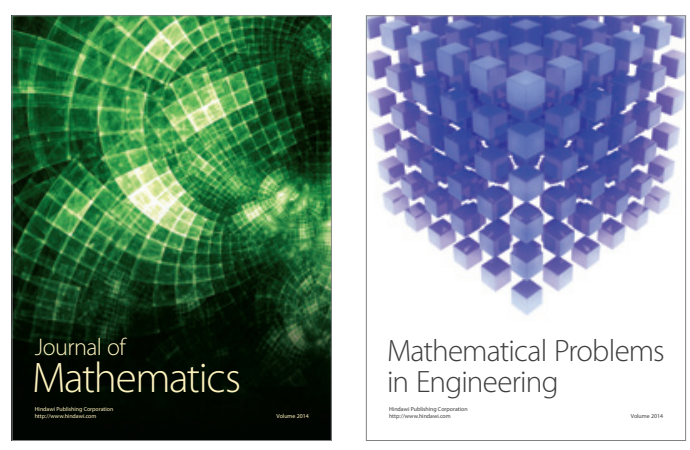

Mathematical Problems in Engineering
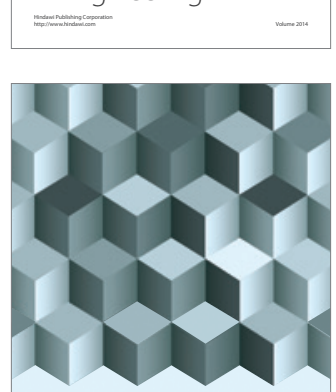

Journal of

Function Spaces
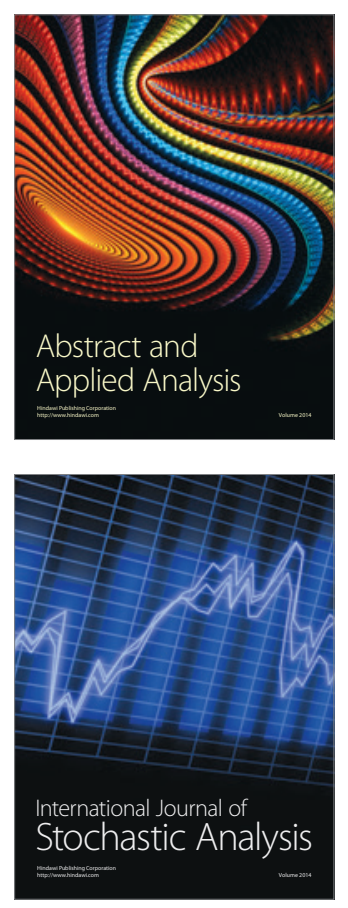

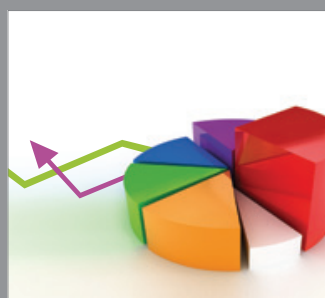

ournal of

Probability and Statistics

Promensencen
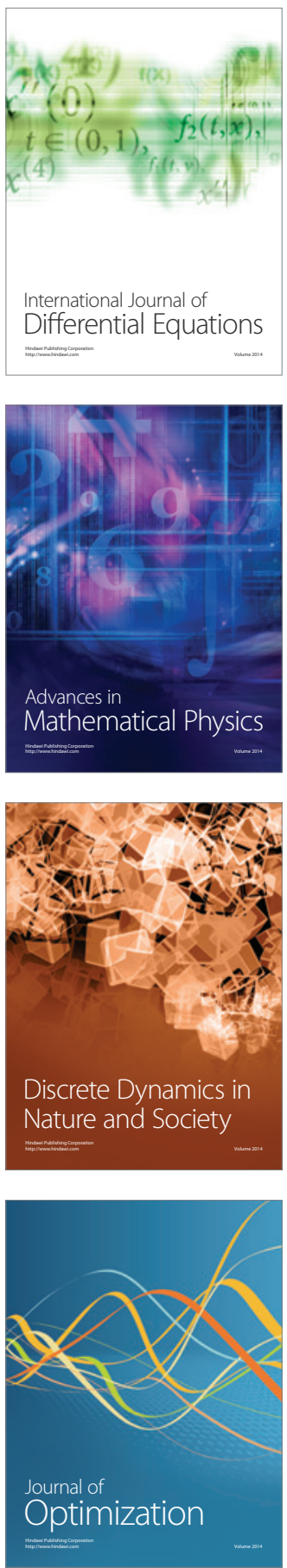